\title{
Acute exacerbation of idiopathic pulmonary fibrosis as the initial presentation of the disease
}

\author{
K. Sakamoto**\#, H. Taniguchi ${ }^{\#}$, Y. Kondoh", K. Ono ${ }^{\star}$, Y. Hasegawa* and M. Kitaichi ${ }^{+}$
}

ABSTRACT: The clinical course of patients with idiopathic pulmonary fibrosis (IPF) is generally marked by a decline in pulmonary function over time, although recently there is increasing recognition that fatal deterioration from acute exacerbation can occur at any stage.

The patient described in the present case study was a 65 -yr-old male who presented with exertional dyspnoea and fever of 2 weeks' duration. He had no history of chronic lung disease or physiological or radiological hallmarks of pre-existing disease. He underwent surgical lung biopsy and the histological examination showed a background pattern of usual interstitial pneumonia (UIP) with a pattern of focal acute diffuse alveolar damage (DAD) in the area where normal lung architecture was preserved.

It is notable that the pathological diagnosis of this rapidly progressive interstitial pneumonia was DAD on UIP, which is typically seen in acute exacerbations of IPF. Unusual findings on highresolution computed tomography scan were also noted.

We presume that in this case acute exacerbation developed in the very early course of IPF. Given the possibility that similar cases may have arisen among patients diagnosed with acute interstitial pneumonia or acute respiratory distress syndrome, the histopathology of rapidly progressive interstitial pneumonia may need to be revisited.

KEYWORDS: Acute exacerbation, diffuse alveolar damage, idiopathic pulmonary fibrosis, rapidly progressive interstitial pneumonia, surgical lung biopsy, usual interstitial pneumonia

$\mathrm{T}$ he concept of acute exacerbation (AE) of idiopathic pulmonary fibrosis (IPF) is increasingly recognised, and findings from several cohorts suggest it is a major cause of death in IPF [1, 2]. Most cases of AE-IPF in previous reports have been characterised by the pathological hallmark of diffuse alveolar damage (DAD) on an underlying fibrotic pattern of usual interstitial pneumonia (UIP) [3-5]. Here, we report a case presenting acute respiratory failure without any chronic respiratory symptoms or the radiological hallmark of chronic fibrotic findings. Nevertheless, video-assisted thoracoscopic surgical lung biopsy of the patient revealed pathological findings of DAD superimposed on a UIP pattern.

\section{CASE REPORT}

A 65-yr-old Japanese male was admitted to Tosei General Hospital (Seto, Japan) in April 2007 with exertional dyspnoea and fever. He had been well until $\sim 2$ weeks earlier, when he developed a sore throat and general fatigue. He visited a local doctor who prescribed $300 \mathrm{mg}$ levofloxacine once daily, but this did not relieve his symptoms. A week before presentation he also noticed dyspnoea on exertion and low-grade fever. He was an ex-smoker with a 36 pack-yr history, and an office worker who denied any history of dust or chemical exposure. There was nothing noteworthy in his family or past history except for glaucoma. On physical examination, the patient appeared to be fatigued. His body temperature was $37.8^{\circ} \mathrm{C}$, cardiac frequency $84 \mathrm{~min}^{-1}$, and blood pressure $130 / 84 \mathrm{mmHg}$. His respiratory frequency was $28 \mathrm{~min}^{-1}$. His larynx was slightly reddish and bibasilar late inspiratory fine crackles were audible in the lungs. No pedal oedema or digital clubbing was observed. No signs of collagen vascular diseases were present. The remainder of the physical examination was normal.
AFFILIATIONS

*Dept of Respiratory Medicine, Nagoya University Graduate School of Medicine, Nagoya,

Depts of ${ }^{\#}$ Respiratory Medicine and Allergy, and

'Pathology, Tosei General Hospital, Seto, and

${ }^{+}$Dept of Laboratory Medicine and Pathology, Kinki-chuo Chest Medical Center, Osaka, Japan.

\section{CORRESPONDENCE}

H. Taniguchi

Dept of Respiratory Medicine and

Allergy

Tosei General Hospital

Seto

Japan

E-mail: taniguchi@tosei.or.jp

Received:

February 162009

Accepted after revision:

February 232009

STATEMENT OF INTEREST

None declared.

PROVENANCE

Submitted article, peer reviewed. 
Chest radiography showed a nonsegmental, peripheral ground-glass opacification predominantly in the upper and middle lung fields. On high-resolution computed tomography (HRCT) of the chest, subpleural reticular opacity superimposed with ground-glass attenuation was recognised with a definable boundary (fig. 1). No traction bronchiectasis, honeycombing or pleural effusion was observed. A chest radiograph taken in a physical check-up 6 months prior to admission was obtained; however, this was completely normal.

Laboratory findings on admission were an increased white blood cell count $\left(11,400 \mathrm{~mm}^{-3}\right)$, elevated C-reactive protein $\left(13.8 \mathrm{mg} \cdot \mathrm{dL}^{-1}\right)$, and slightly elevated lactate dehydrogenase. The level of serum KL-6 was slightly elevated $\left(762 \mathrm{U} \cdot \mathrm{mL}^{-1}\right)$.

Pulmonary function tests showed normal spirometry but decreased gas diffusion capacity (vital capacity 99.1\% predicted, diffusing capacity of the lung for carbon monoxide $58.4 \%$ pred). The lymphocyte stimulation test for levofloxacin was negative.
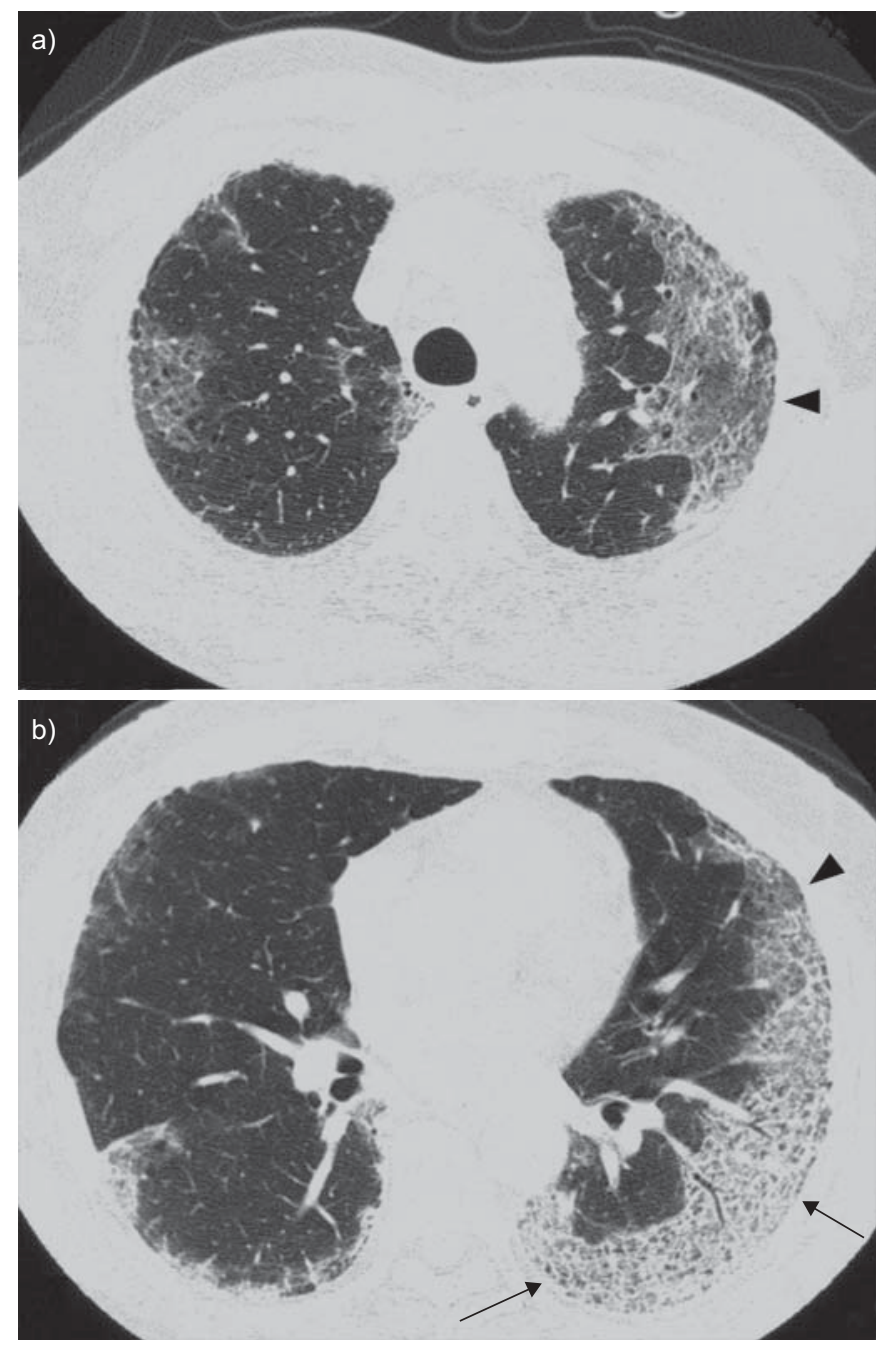

FIGURE 1. High-resolution computed tomography of the chest obtained on admission shows ground-glass opacity (arrowheads), partly overlying abnormal linear opacity (arrows), predominantly affecting the subpleural area. No honeycombing or traction bronchiectasis was observed.
Bronchoalveolar lavage (BAL) performed for the differential diagnosis revealed increased total cell count $\left(800,000 \mathrm{~mm}^{-3}\right)$, lymphocytes $17 \%$, and neutrophils $54 \%$, but no bacterial or viral pathogen was identified from BAL fluid.

After admission, broad-spectrum beta-lactam was administered in place of the discontinued levofloxacin. The patient's chest radiographic findings deteriorated and he became hypoxaemic. To establish histopathological diagnosis, surgical lung biopsy was performed on the seventh day of hospital stay.

Two specimens were obtained from the upper and the lower lobes of the left lung by videothoracoscopic biopsy. Histological examination showed a background pattern of UIP with fibrotic change predominantly distributed in the subpleural and perilobular areas, and an abrupt transition between almost-normal alveolar septum and dense fibrosis with architectural disruption (fig. 2). Additionally, a focal acute DAD pattern was observed with the hyaline membrane in the area where normal lung architecture was preserved.

After biopsy, intravenous high-dose corticosteroids and cyclosporine were administered. Immunosuppressive therapy led to a transient improvement in the respiratory failure, but did not induce remission. The patient had complications with disseminated intravascular coagulopathy and acute renal failure. Finally, on the 44th day in hospital, the patient died of multiple organ failure despite $>30$ days of intensive care.

\section{DISCUSSION}

Here, we have described a case presenting refractory respiratory failure with acute onset. Pathological examination revealed DAD with a background UIP pattern. It is presumed that this patient suffered from a severe AE in the very early course of IPF, which was not identified clinically. An unusual distribution of abnormal opacity on HRCT was also noted.

To eliminate acute lung injury of known cause such as infection and drug-induced lung injury, exploration was performed to the extent feasible. We believe this case was within the spectrum of idiopathic interstitial pneumonias. It has been generally accepted that the differential diagnosis of idiopathic interstitial pneumonias presenting an acute clinical course includes acute interstitial pneumonia (AIP) [6] and cryptogenic organising pneumonia [7]. On the other hand, the pathological finding of DAD overlapping a UIP pattern is typically observed in the setting of AE-IPF [3-5], which usually has a chronic clinical course prior to an acute deterioration phase. The significant features of the present case were as follows: 1) despite the pathological feature of coexisting UIP pattern, there were no "clues" for chronicity, either in history and physical findings or on HRCT images; and 2) the characteristics of the HRCT findings seemed distinctly different from previously reported radiological findings of AIP or AE-IPF.

With respect to AE-IPF without previous diagnosis, PARAMBIL et al. [8] reported seven cases of AE-IPF with pathological confirmation, three of which had no prior diagnosis of IPF. These cases had some small clues as to disease chronicity, such as clubbed fingers and honeycombing on HRCT. KIM et al. [5] reported a similar case. With reference to these reports, a perspective working group proposed criteria for diagnosis of 

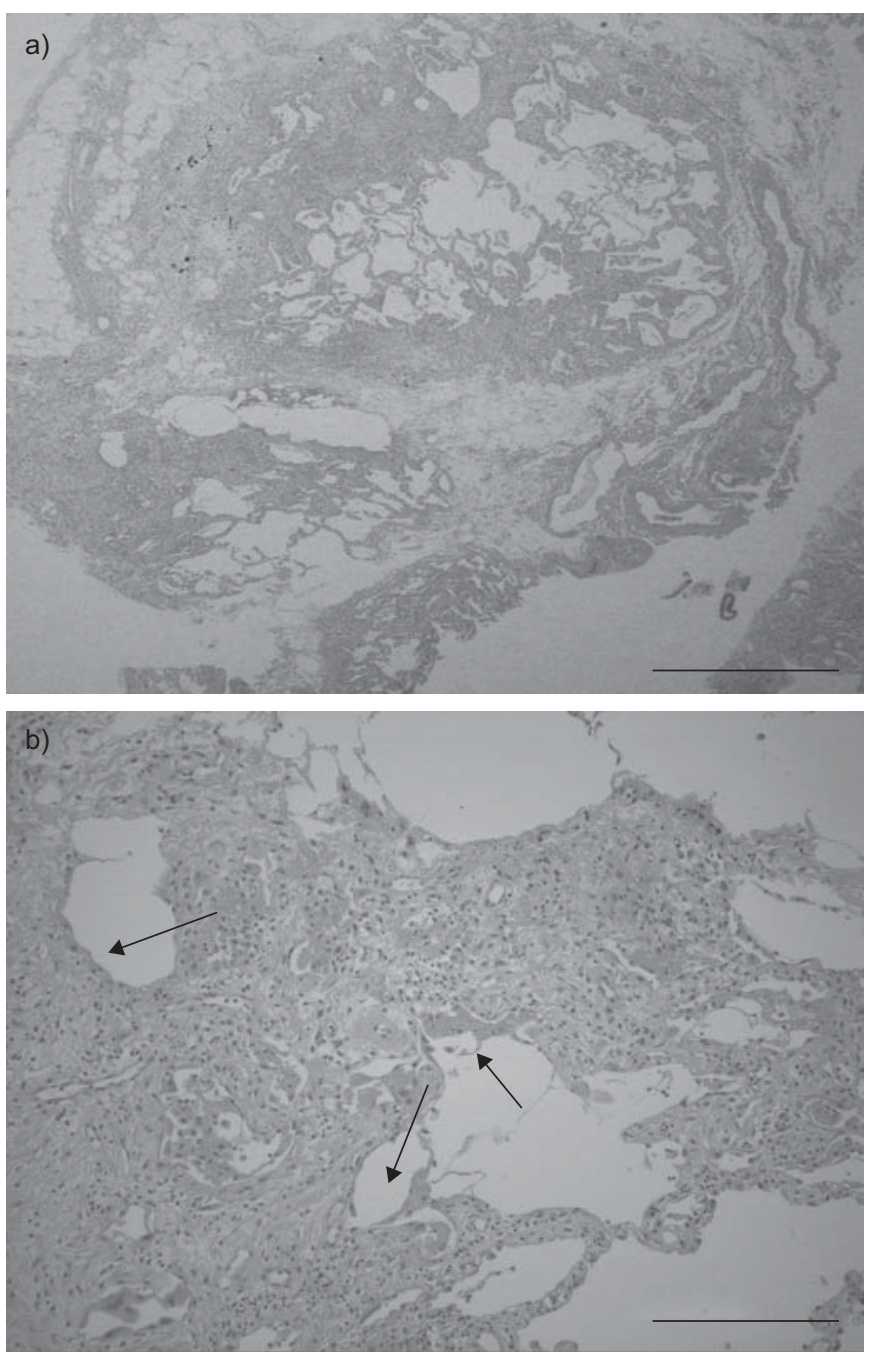

FIGURE 2. Histopathology of the biopsy specimen obtained from the right lower lobe (haematoxylin-eosin stain). a) Low-magnification photomicrograph showing a background of fibrosis and honeycomb change typical of usual interstitial pneumonia with an area of associated diffuse alveolar damage (DAD) at upper centre. Scale bar=1 mm. b) Higher magnification of area of acute DAD. Proliferating spindle cells expand and distort alveolar septa with hyaline membranes and hyalinous exudates (arrows). Scale bar $=200 \mu \mathrm{m}$.

AE-IPF [9]. According to these criteria, AE-IPF can be diagnosed by the presence of a background reticular or honeycomb pattern consistent with UIP pattern if a diagnosis of IPF has not been previously established. In this case, no fibrotic hallmark of honeycombing, traction bronchiectasis, or architectural distortion was found on HRCT, so the presence of pathological UIP pattern could not be predicted. In this case, an AE may have occurred very early in the course of IPF, since the pathological features of UIP were observed despite the absence of the radiological hallmarks of UIP. Assessment of the appropriateness of diagnosing such cases as AE-IPF is warranted. For instance, if similar cases can be rescued, it should be confirmed whether their clinical courses are compatible with IPF.

On HRCT, an unusual patchy peripheral distribution of ground-glass opacity with reticulation was observed. This is not typical of DAD as more classically described in either AIP or IPF. AKIRA et al. [10] reported an association between distribution of HRCT abnormality and pathological findings in a recent case series. Multifocal and diffuse opacity corresponded to DAD, whereas peripheral opacity corresponded to active fibrotic foci. The observations in the present case differ from previous reports. The interpretation of the unusual distribution in this case is difficult. Typical changes of UIP tend to spread from the peripheral areas of the lung in IPF. DAD in this case appears to extend from areas where early UIP change is supposed to be established. This suggests that an anatomical abnormality resulting from UIP (such as disruption of lymphatic channels) might have predisposed the patient to the problem, or might have made the problem more obvious on HRCT in that region. From the fact that underlying UIP change and alveolar damage were observed together in the same pathological slide, the alveolar damage seems to have extended to the preserved area adjacent to established UIP. We believe the DAD changes occurred in the adjacent lesion where underlying UIP changes existed at the time of HRCT, and subsequently spread extensively.

In our institution (Tosei General Hospital, Seto, Japan), we have been taking an aggressive diagnostic approach including surgical lung biopsy in selective cases with acute respiratory failure of unknown origin, although the role of lung biopsy in patients with acute respiratory failure has been questioned due to potentially high morbidity and low diagnostic yield. Recent studies have shown, however, that surgical lung biopsy in acute respiratory distress syndrome can be done safely and has contributed to treatment changes that led to better outcomes $[11,12]$. Moreover, SuH et al. [13] reported favourable outcomes in AIP patients as a result of rapid diagnosis by surgical lung biopsy together with early initiation of immunosuppressants. In addition, KONDOH et al. studied pathological findings in 28 patients with rapidly progressive interstitial pneumonia and found that the presence of DAD or DAD with UIP on lung biopsy specimen is a marker of worse prognosis than that of organising pneumonia or nonspecific interstitial pneumonia (Y. Kondoh, H. Taniguchi, K. Kataoka, et al., Tosei General Hospital; personal communication). Although we do not have sufficient data to demonstrate the necessity of surgical lung biopsy to differentiate DAD from DAD with UIP, we think that in selected patients with acute respiratory failure of unknown cause, an aggressive diagnostic approach including surgical lung biopsy may be useful.

We suggest that previous cases diagnosed as AIP or acute respiratory distress syndrome might have included cases of $\mathrm{AE}$ of early IPF similar to the present case. The histopathology of rapidly progressive interstitial pneumonia may need to be revisited in this context.

\section{REFERENCES}

1 Azuma A, Nukiwa T, Tsuboi E, et al. Double-blind, placebo-controlled trial of pirfenidone in patients with idiopathic pulmonary fibrosis. Am J Respir Crit Care Med 2005; 171: 1040-1047.

2 Martinez FJ, Safrin S, Weycker D, et al. The clinical course of patients with idiopathic pulmonary fibrosis. Ann Intern Med 2005; 142: 963-967. 
3 Kondoh Y, Taniguchi H, Kawabata Y, et al. Acute exacerbation in idiopathic pulmonary fibrosis. Analysis of clinical and pathologic findings in three cases. Chest 1993; 103: 1808-1812.

4 Ambrosini V, Cancellieri A, Chilosi M, et al. Acute exacerbation of idiopathic pulmonary fibrosis: report of a series. Eur Respir J 2003; 22: 821-826.

5 Kim DS, Park JH, Park BK, et al. Acute exacerbation of idiopathic pulmonary fibrosis: frequency and clinical features. Eur Respir J 2006; 27: 143-150.

6 Bouros D, Nicholson AC, Polychronopoulos V, et al. Acute interstitial pneumonia. Eur Respir J 2000; 15: 412-418.

7 Cordier JF. Cryptogenic organising pneumonia. Eur Respir J 2006; 28: 422-446.

8 Parambil JG, Myers JL, Ryu JH. Histopathologic features and outcome of patients with acute exacerbation of idiopathic pulmonary fibrosis undergoing surgical lung biopsy. Chest 2005; 128: 3310-3315.

9 Collard HR, Moore BB, Flaherty KR, et al. Acute exacerbations of idiopathic pulmonary fibrosis. Am J Respir Crit Care Med 2007; 176: 636-643.

10 Akira M, Kozuka T, Yamamoto S, et al. Computed tomography findings in acute exacerbation of idiopathic pulmonary fibrosis. Am J Respir Crit Care Med 2008; 178: 372-378.

11 Patel SR, Karmpaliotis D, Ayas NT, et al. The role of openlung biopsy in ARDS. Chest 2004; 125: 197-202.

12 Papazian L, Doddoli C, Chetaille B, et al. A contributive result of open-lung biopsy improves survival in acute respiratory distress syndrome patients. Crit Care Med 2007; 35: 755-762.

13 Suh GY, Kang EH, Chung MP, et al. Early intervention can improve clinical outcome of acute interstitial pneumonia. Chest 2006; 129: 753-761. 